\title{
420 fs Pulses from an Ultrafast Laser Inscribed Waveguide Laser Utilizing a Carbon Nanotube Saturable Absorber
}

\author{
S. J. Beecher, R. R. Thomson, N. D. Psaila and A. K. Kar \\ School of Engineering and Physical Sciences, Heriot-Watt University, Edinburgh, EH14 4AS, Scotland. \\ sb215@hw.ac.uk,+44(0)1314513648 \\ Z. Sun, T. Hasan, A. G. Rozhin and A. Ferrari \\ Engineering Department, University of Cambridge, Cambridge CB3 OFA, United Kingdom
}

\begin{abstract}
We report the generation of $420 \mathrm{fs}$ pulses of $1.56 \mu \mathrm{m}$ light from a mode-locked ultrafast laser inscribed Er-doped waveguide laser. Passive mode-locking was achieved using a carbon nanotube saturable absorber.
\end{abstract}

(C)2010 Optical Society of America

OCIS codes: (130.3120) Integrated optics devices; (140.3500) Lasers, erbium; (140.3390) Laser materials processing

\section{Introduction}

The use of ultrafast lasers for inscribing structural modifications in transparent materials is currently attracting considerable research attention [1]. One application area of ultrafast laser inscription (ULI) that has attracted particular attention is the fabrication of active optical waveguide devices such as waveguide amplifiers [2,3], and waveguide lasers $[4,5]$. Given the success of ULI in these areas, there is now a drive to push the boundaries of what can be achieved. A number of groups are now working to increase output powers, demonstrate new wavelengths and pulsed operation using Q-switching and mode-locking techniques. To date however, there has only been one report of successful mode-locking of a ULI fabricated waveguide laser [4], and the authors were only able to demonstrate $1.6 \mathrm{ps}$ pulses due to the low round trip net gain of their laser cavity. Here we report the generation of femtosecond pulses from a mode-locked ULI fabricated waveguide laser. This demonstration has been enabled by the realization of a high-gain ULI fabricated Er-doped bismuthate glass waveguide amplifier [3], and the use of a well developed carbon nanotube (CNT) saturable absorber [6-10].

\section{Waveguide amplifier development}

The glass substrate used in this study was an Er-doped bismuthate glass containing $>70 \mathrm{wt} . \% \mathrm{Bi}_{2} \mathrm{O}_{3}$ and doped with 0.63 wt. \%. Er. The glass was supplied by AGC in Japan. Waveguides were inscribed using a custom designed, variable repetition-rate master-oscillator power-amplifier (MOPA) ultrafast fiber laser system supplied by Fianium Ltd. For this work, the repetition-rate of the laser was set to $500 \mathrm{kHz}$ and the pulse duration was measured to be $\approx$ $350 \mathrm{fs}$, full-width at half maximum. The central wavelength of the laser radiation was $1064 \mathrm{~nm}$ and the polarization was adjusted to be circular. The pulse train was focused into the sample using a 0.4 NA aspheric lens and the sample was translated through the focus using computer controlled $x-y-z$ air-bearing stages (Aerotech). The waveguide cross section was controlled using the multiscan fabrication technique [3]. As a consequence, each waveguide was fabricated using 20 scans of the substrate through the laser focus, with each scan separated by $0.4 \mu \mathrm{m}$ in the axis perpendicular to both the waveguide axis and the laser beam propagation axis. The optimum waveguide was inscribed using $133 \mathrm{~nJ}$ pulses and a translation speed of $1.0 \mathrm{~mm} \cdot \mathrm{s}^{-1}$. After inscription, the sample was ground and polished at an angle of $3.0^{\circ}$ to the waveguide axis, in order to reduce back-reflections. The final waveguide length was $87.0 \mathrm{~mm}$.

Prior to constructing a laser cavity, the performance of the optimum waveguide was characterized. When two sections of 980/1550 nm wavelength division multiplexer (WDM) coupler fiber were butt-coupled to the waveguide facets (using index matching gel to reduce Fresnel reflections) the waveguide amplifier exhibited a background insertion loss of $4.2 \mathrm{~dB}$, measured outside the Er-ion absorption band at $1620 \mathrm{~nm}$, and a polarization dependent loss of $\approx 0.3 \mathrm{~dB}$ at $1533 \mathrm{~nm}$. When pumped with $\approx 1.0 \mathrm{~W}$ of $980 \mathrm{~nm}$ light, the waveguide amplifier exhibited a peak fiber-to-fiber net gain of $\approx 16.0 \mathrm{~dB}$ at $1533.0 \mathrm{~nm}$, and greater than $10.0 \mathrm{~dB}$ of net gain from $1527 \mathrm{~nm}$ to $1563 \mathrm{~nm}$. 


\section{CThI6.pdf}

\section{Mode-locked waveguide laser construction and operation}

A fiber ring cavity was constructed around the Er-doped bismuthate waveguide amplifier, as shown in Fig. 1(a). The optical isolator ensures unidirectional operation, thus reducing instabilities caused by reflections. Mode-locking is initiated by a CNT-polymer composite saturable absorber, which is sandwiched between 2 FC/PC fiber connectors. Output coupling is achieved using a broadband 90:10 fused fiber coupler. The pump light is delivered to the waveguide amplifier via two fused fiber wavelength division multiplexers (WDMs).
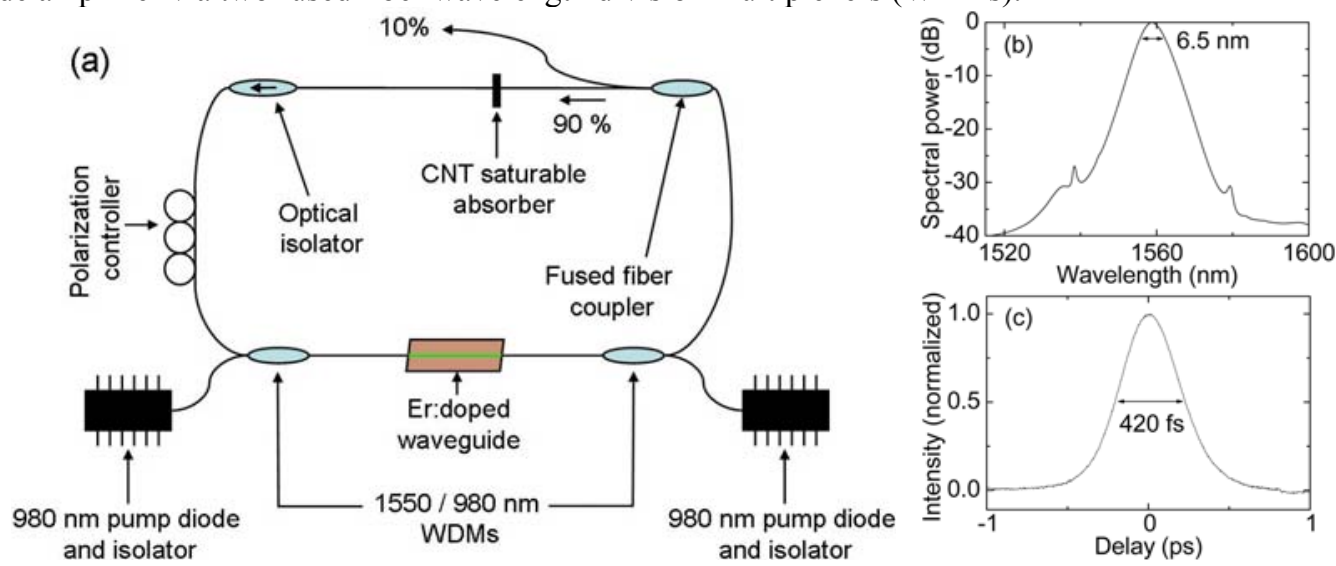

Figure 1 (a) Schematic diagram of the mode-locked waveguide laser cavity. (b) Optical spectrum of the mode-locked laser output (c) An autocorrelation of the output from the mode-locked laser assuming a sech ${ }^{2}$ pulse shape.

Continuous wave $(\mathrm{CW})$ oscillation started upon the application of $220 \mathrm{~mW}$ of counter-propagating pump power and $70 \mathrm{~mW}$ of co-propagating pump power. Self-starting single-pulse mode-locking initiated when the co-propagating pump was increased to $80 \mathrm{mw}$, with no Q-switching instabilities observed. The average output power increased with co-propagating pump power up to $0.5 \mathrm{~mW}$ of mode-locked output for $105 \mathrm{~mW}$ of co-propagating and $220 \mathrm{~mW}$ counter-propagating pump power. Although considerably more pump power was available, we did not attempt to exceed the $0.5 \mathrm{~mW}$ output power to avoid damage to the CNT-polymer composite. Fig. 1(b) plots the output optical spectrum with central wavelength $\approx 1560 \mathrm{~nm}$ and full-width at half maximum bandwidth of $6.5 \mathrm{~nm}$. As shown in Fig. 1(c), an autocorrelation measurement yielded a pulse duration of $420 \mathrm{fs}$, assuming a sech ${ }^{2}$ temporal profile, close to the bandwidth limit of 395 fs. A fast photo-diode and RF spectrum analyzer confirmed pulsing at a repetition rate of $39 \mathrm{MHz}$.

\section{Conclusions}

We have demonstrated a ULI fabricated, mode-locked waveguide laser producing $420 \mathrm{fs}$ pulses with a full-width at half maximum spectral width of $6.5 \mathrm{~nm}$. We believe this work will pave the way to compact, high-efficiency and high repetition-rate mode-locked lasers using the two novel technologies of ULI and CNT based saturable absorbers.

\section{References}

[1] K. M. Davis et al., "Writing waveguides in glass with a femtosecond laser," Opt. Lett. 21, 1729-1731 (1996)

[2] G. Della Valle, et al., "C-band waveguide amplifier produced by femtosecond laser writing," Opt. Express 13, 5976-5982 (2005)

[3] R. R. Thomson et al. " Ultrafast Laser Inscription of a High Gain Er-doped Bismuthate Glass Waveguide Amplifier," in Advanced SolidState Photonics, OSA Technical Digest Series (CD) (Optical Society of America, 2010), paper AWB7.

[4] G. Della Valle et al., "Passive mode locking by carbon nanotubes in a femtosecond laser written waveguide laser," Appl. Phys. Lett. 89, 231115 (2006)

[5] F. M. Bain et al., "Ultrafast laser inscribed $\mathrm{Yb}: \mathrm{KGd}\left(\mathrm{WO}_{4}\right)_{2}$ and $\mathrm{Yb}: \mathrm{KY}\left(\mathrm{WO}_{4}\right)_{2}$ channel waveguide lasers," Opt. Express 17, 22417-22422 (2009)

[6] F. Wang et al., "Wideband-tuneable, nanotube mode-locked, fibre laser," Nat. Nanotechnol. 3, 738-742 (2008).

[7] V. Scardaci et al., "Carbon Nanotube Polycarbonate Composites for Ultrafast Lasers." Adv. Mater. 20, 4040-4043 (2008).

[8] E. J. R. Kelleher et al., "Nanosecond-pulse fiber lasers mode-locked with nanotubes," Appl. Phys. Lett. 95, 111108 (2009).

[9] Z. Sun et al., "L-band ultrafast fiber laser mode locked by carbon nanotubes." Appl. Phys. Lett. 93, 061114 (2008).

[10] T. Hasan et al., "Nanotube-Polymer Composites for Ultrafast Photonics," Adv. Mater. 21, 3874-3899 (2009).

\section{Acknowledgements}

This work was funded by the UK Engineering and Physical Sciences Research Council (EPSRC) (Grant Nos. EP/D047269/1, EP/G030227/1 and EP/GO30480/1), the Royal Society, European Research Council Grant NANOPOTs and King's College, Cambridge. We sincerely thank Fianium Ltd. for their support and AGC-Japan for supplying the Er-doped bismuthate glass used in this study. 